\title{
Multi-Functions of Net Surface Charge in the Reaction on a Single Nanoparticle
}

\author{
Shaobo $\mathrm{Xi}^{1}$ and Xiaochun Zhou* ${ }^{1,2}$ \\ ${ }^{1}$ Division of Advanced Nanomaterials, ${ }^{2}$ Key Laboratory of Nanodevices and Applications, Suzhou \\ Institute of Nano-tech and Nano-bionics, Chinese Academy of Sciences, Suzhou 215125, China.
}

\section{Table of Contents}

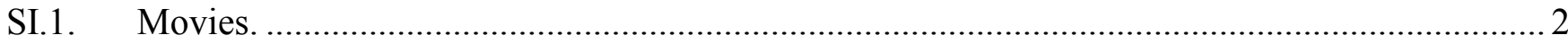

SI.2. Characterizations of Ag Nanowires Before and After GRR............................................... 2

SI.3. Drift Correction by Super Resolution microscopy. ........................................................ 3

SI.4. Calculating the Intensity Trajectory versus Time along the Ag Nanowire.............................. 4

SI.5. Reaction Rate along a Single Ag Nanowire Controlled by Electric Field............................... 6

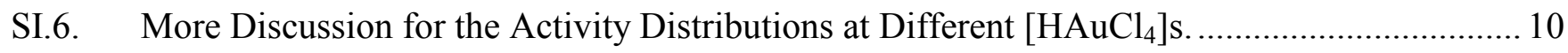




\section{SI.1. Movies.}

Movie S1: Dark-field movie of many single Ag nanowires in the GRR at low concentration of $\mathrm{HAuCl}_{4}$ during the time from 0 to $\sim 5 \mathrm{ks}$.

Movie S2: Dark-field movie of many single Ag nanowires in the GRR at high concentration of $\mathrm{HAuCl}_{4}$ during the time from 0 to $\sim 2 \mathrm{ks}$.

\section{SI.2. Characterizations of Ag Nanowires Before and After GRR.}

The as-synthesized Ag nanowires were characterized by TEM, SEM in Figure S1. The TEM and SEM images in Figure S1A-B shows that the Ag nanowires are of tens nm diameter and very long length. Figure S1C-D shows that the diameter and the length of $\mathrm{Ag}$ nanowires are $50.1 \pm 8.5 \mathrm{~nm}$ and $6.3 \pm 3.6 \mu \mathrm{m}$.
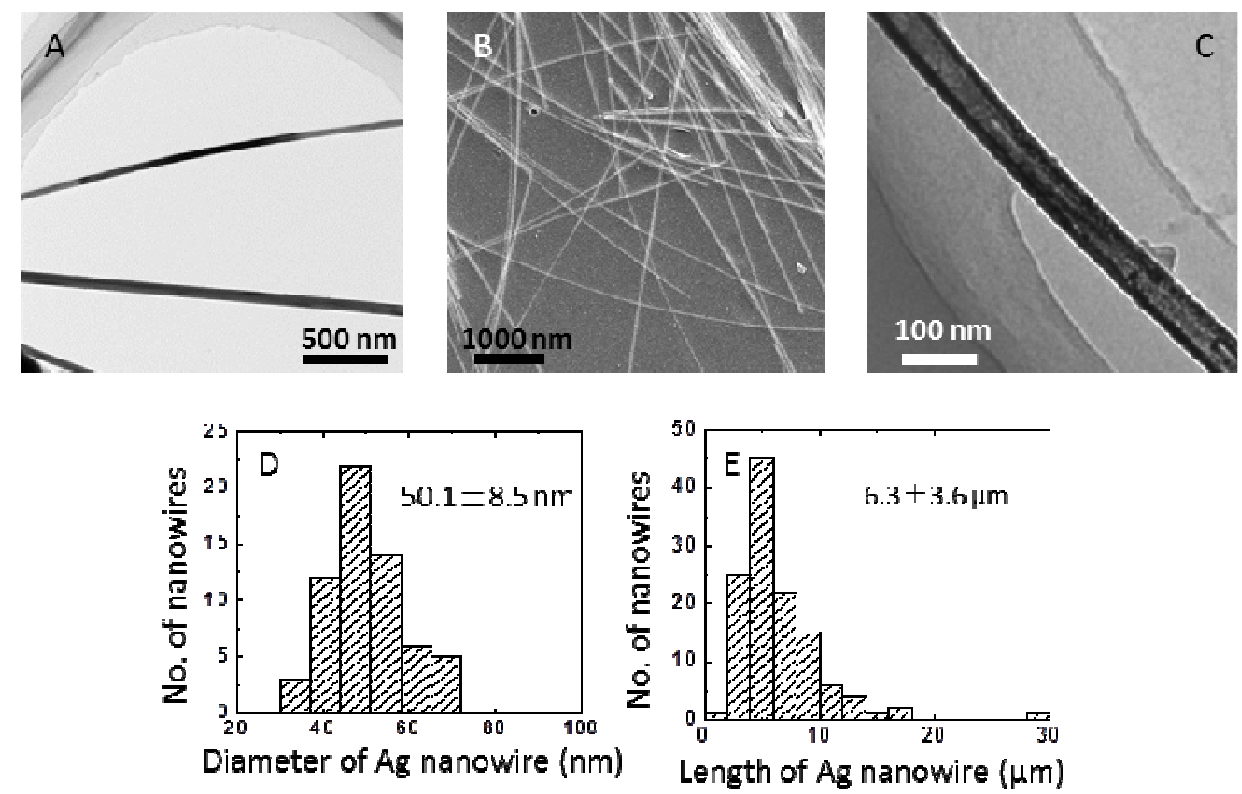

Figure S1. (A)-(B) TEM and SEM images of as synthesized Ag nanowires. (C) TEM image of Ag nanowire after GRR at $0.02 \mathrm{mM} \mathrm{HAuCl}_{4}$. (D)-(E) Diameter and Length of as synthesized Ag nanowires. 

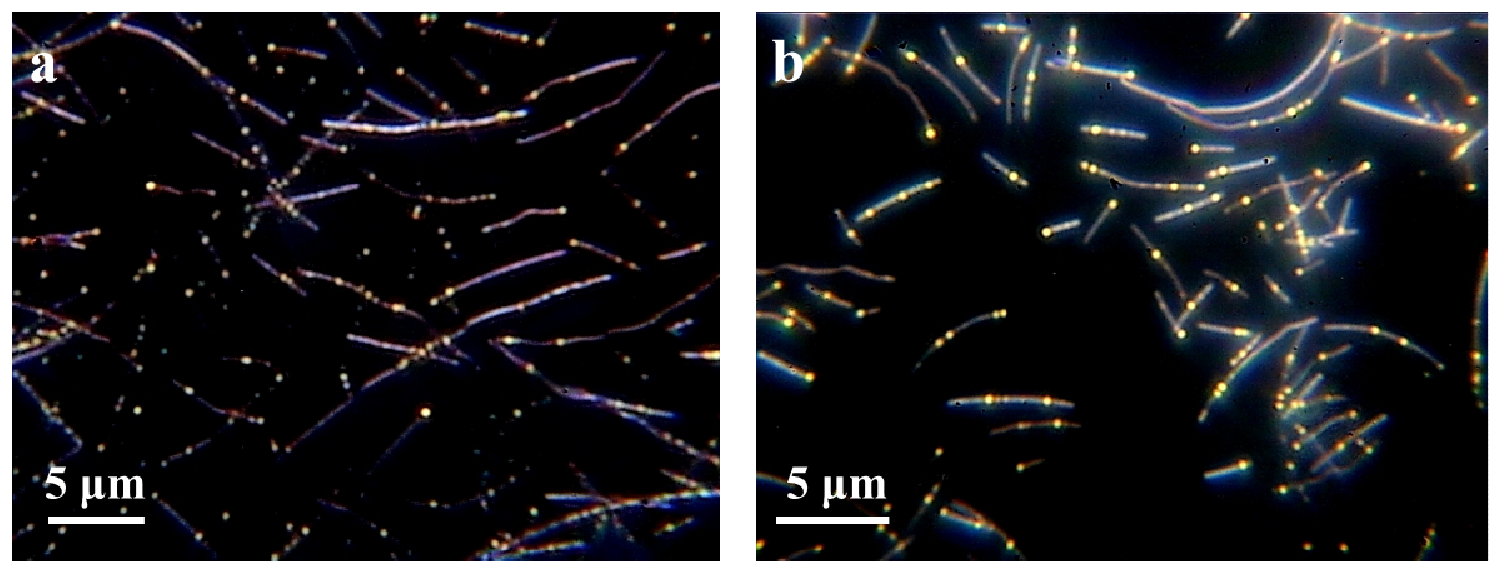

Figure S 2. Dark-field image of well dispersed single Ag nanowires taken by color camera after $2.4 \mathrm{ks}$ GRR at $0.02 \mathrm{mM}\left[\mathrm{HAuCl}_{4}\right]$.
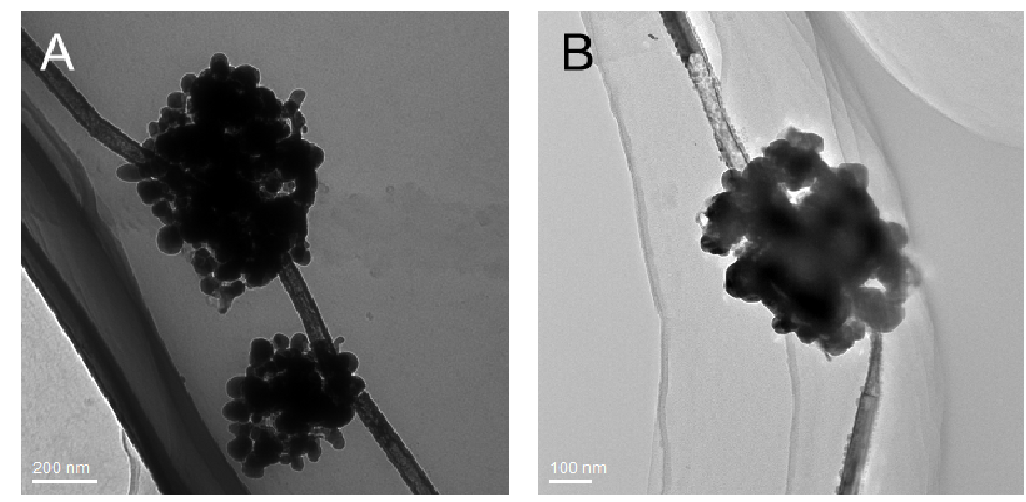

Figure S 3. More TEM images of hot spots generated at $0.02 \mathrm{mM}\left[\mathrm{HAuCl}_{4}\right]$.

\section{SI.3. Drift Correction by Super Resolution microscopy.}
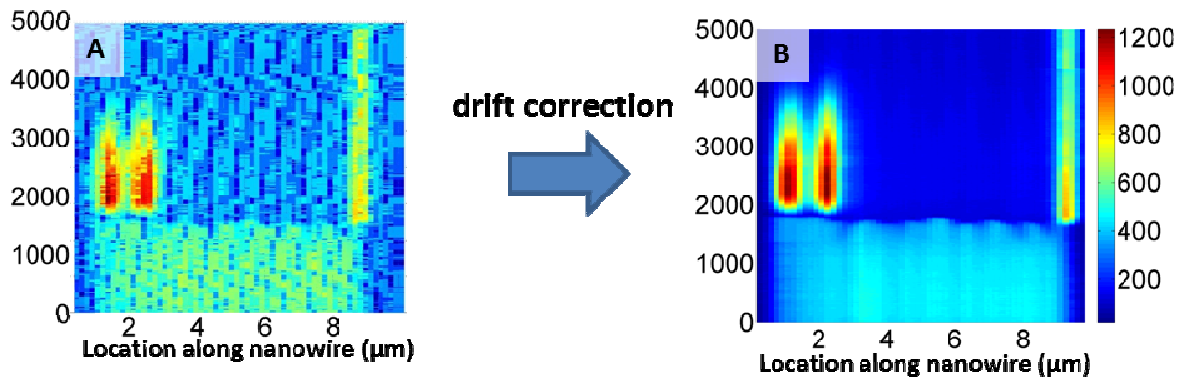

Figure S 4. Real examples for the drift correction. (A) 2D light scattering intensity versus time along the Ag nanowire before the drift correction. (B) After the drift correction. 


\section{SI.4. Calculating the Intensity Trajectory versus Time along the Ag Nanowire.}

In order to study the kinetics of each location of the single Ag nanowires, the intensity trajectory versus time along the whole Ag nanowire needs to be calculated. The calculation includes four steps, which will be introduced as follows.

Step1, drift correction was done for each frame in the movie before the scattering intensity calculation, Figure S 5B .

Step2, the well isolated Ag nanowires, which were single nanowires, were selected out from the image. (Figure S 5C).

Step3, the single Ag nanowire was segmented into a number of segments with the width of one pixel shown in Figure S 5D with yellow rectangular. For the straight nanowire, the axis of the nanowire was determined by the linear fitting. Then the nanowire was segmented into a number of segments along the axis. For the curving nanowire, tangent line (red line in Figure S 5D) along the nanowire was calculated first. Then the nanowire was segmented into a number of segments every one pixel along the tangent line shown in Figure S 5E.

Step4, the scattering intensity of each segment was integrated together to get the trajectory versus time. When the intensity trajectories of all segments along the nanowire were plotted, a 2D picture will be obtained Figure 2 and Figure S 5F. This picture can be used to do further kinetics analysis. 
Supplementary Information
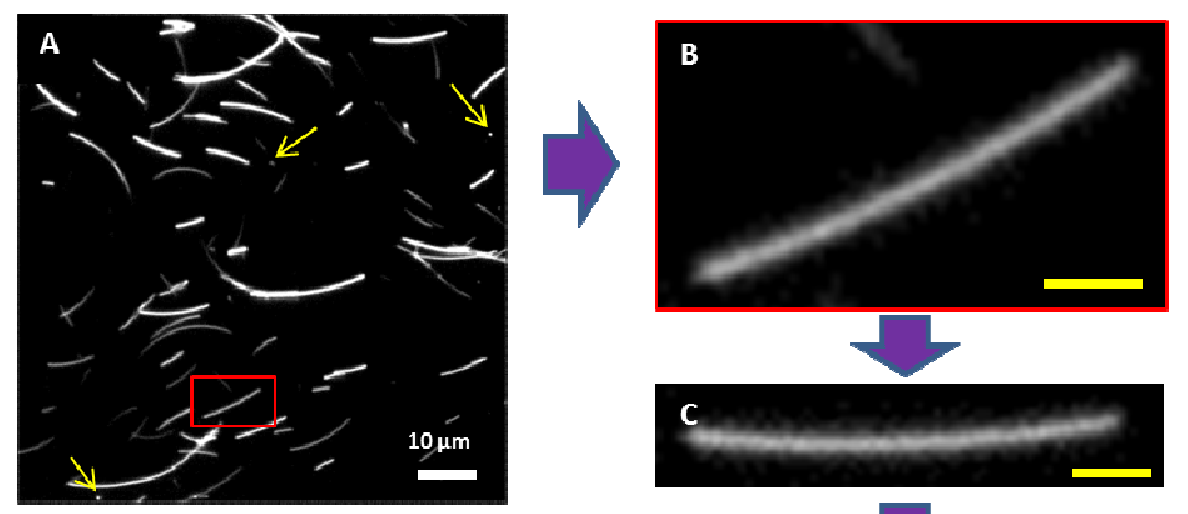

step1

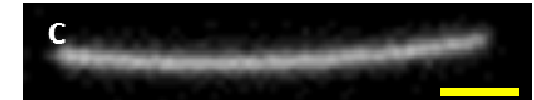

step2

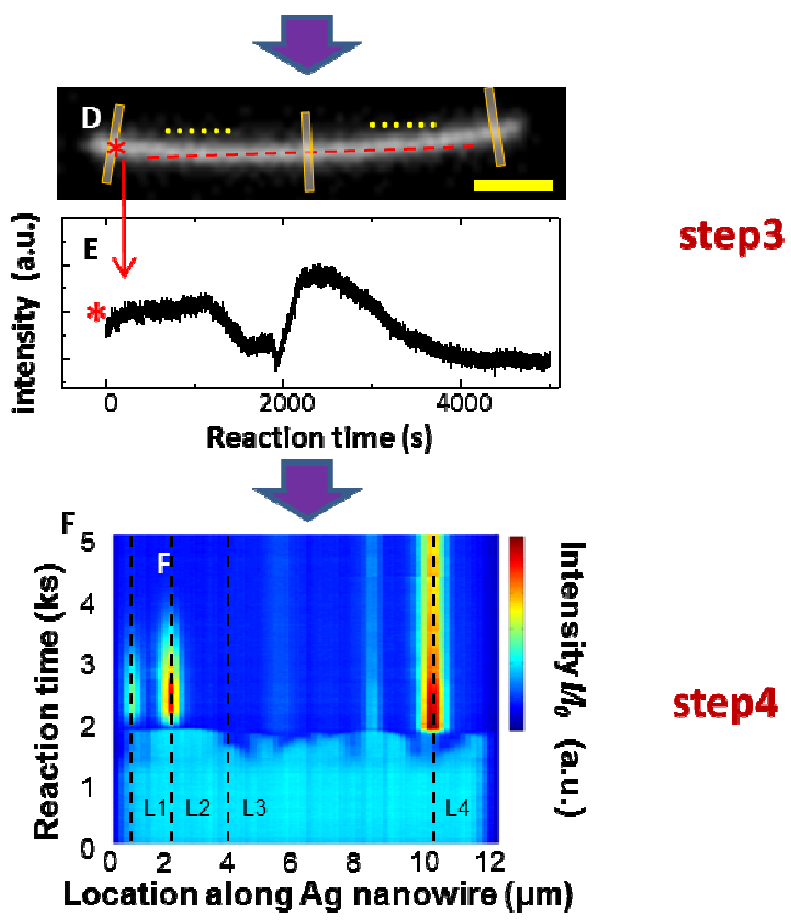

Figure S 5. Calculating the intensity trajectory versus time along the Ag nanowire. (A) Original darkfield image, the Ag nanowire in red rectangular is what we plan to study. (B) The nanowire after drift correction. (C) The nanowire was isolated, and rotated. (D) The nanowire was segmented to segments along the nanowire. (E) Single trajectory from one segment. (F) 2D picture made by the intensity trajectories of all segments along the nanowire. The scale bar in (B-D) is $2 \mu \mathrm{m}$. 


\section{SI.5. Reaction Rate along a Single Ag Nanowire Controlled by Electric Field}

The scattering intensity detected by camera is composed of two parts as shown in Figure S 6. One is the scattering intensity from the deposition of $\mathrm{Ag}$ or $\mathrm{Au}$ (green curve), and another is the scattering intensity from the body of nanowire (yellow curve). The scattering intensity from the body of nanowire decreases with GRR to $I_{1}$, while the scattering intensity from the deposition of Ag or Au increases to $I_{2}$. The total scattering intensity detected by camera will go to $I_{3}$. So

$$
I_{3}=I_{1}+I_{2}
$$

If $I_{1}<<I_{1}$, then

$$
I_{3} \approx I_{2}
$$

The reaction rate is

$$
r^{\prime}=\frac{\Delta I}{\Delta t} \approx \frac{I_{2}}{\Delta t}=\frac{I_{3}}{\Delta t}
$$

In order to measure the reaction rate, we calculate the intensity in the $2 \mathrm{D}$ plot of scattering intensity from the start to the end of reaction.

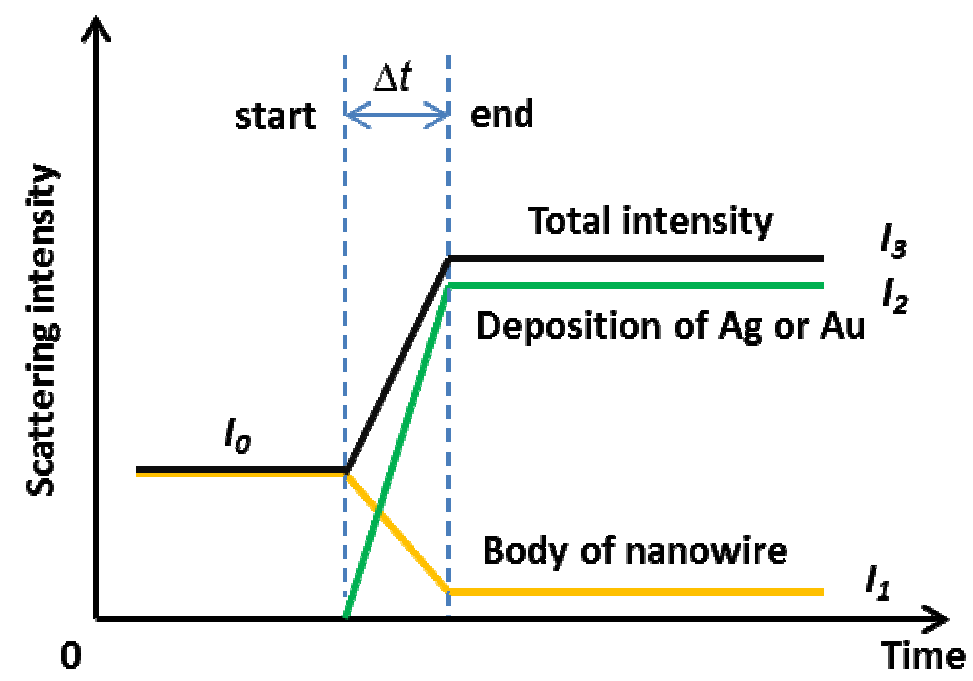

Figure S 6. Composition of scattering intensity at one location of nanowire. The black curve is the total intensity. The green curve is the scattering intensity from the deposition of Ag or Au. The yellow curve is the scattering intensity from the body of nanowire. 
The experiment (e.g. Figure 2 in main text) shows that the growth of bright spots always happens with the abrupt decrease of scattering intensity along the whole nanowire. The rapid decrease of intensity may be due to the reaction of a highly active critical intermediate ${ }^{1}$. The potential trajectories of Ag nanowires on gold electrode during GRR at different $\left[\mathrm{HAuCl}_{4}\right]$ show that the trajectories always have a potential dropping. The potential dropping implies that the Ag nanowires are injected a lot of electrons ${ }^{2}$ through the two reactions,

$$
\begin{aligned}
& A g-e^{-} \rightarrow \mathrm{Ag}^{+} \\
& A g+C l^{-}-e^{-} \rightarrow A g C l
\end{aligned}
$$

A lot of electrons will be injected into the $\mathrm{Ag}$ nanowire to facilitate the reduction of $\mathrm{Ag}^{+}$or $\mathrm{AuCl}_{4}{ }^{-}$. Though a double electric layer will form to neutralize the electrons, excess electrons also exist to make the Ag nanowire work as a cathode electrode. Since the excess electrons will repel each other, the electron distribution on the two ends will be higher than the center. The net charges on the surface of nanomaterial can generate a static electric field around the nanomaterial, which causes a field-induced mass transport ${ }^{3,4}$, and affects the chemical reactions on the surface of nanomaterial. Moreover, high density of net charges can induce the activity variation of nanomaterial ${ }^{5,}{ }^{6}$. Surprisingly and unfortunately, the analytic expression of the charge distribution $\sigma(x)$ on a needle is not available till now $^{7}$. We only find an empirical expression ${ }^{7}$,

$$
\sigma(x)=A+\frac{B}{\left(a^{2}-x^{2}\right)^{1 / 3}}
$$

Where A and B are constants, $a$ is the half length of the needle. Figure S 7A shows the shape of the charge distribution $(\mathrm{A}=0.384985, \mathrm{~B}=0.083684, \mathrm{a}=3 \mathrm{e}-6 \mathrm{~m})^{7}$, which is higher at the two ends. Based on the relation in Figure $\mathrm{S} 7 \mathrm{~B}$, the intensity of the electric field in y direction will be, 


$$
\begin{aligned}
& E_{y}(x)=\int_{-a}^{a} \frac{H \sigma(t) d t}{4 \pi \varepsilon \varepsilon_{0} r^{3}}=\frac{H}{4 \pi \varepsilon \varepsilon_{0}} \int_{-a}^{a} \frac{\sigma(t) d t}{r^{3}} \\
& E_{y}(x)=\frac{H}{4 \pi \varepsilon \varepsilon_{0}} \int_{-a}^{a} \frac{\sigma(t) d t}{\left((t-x)^{2}+H^{2}\right)^{3 / 2}}
\end{aligned}
$$

Since the charge density at the two ends is higher, the electric field in y direction will be higher at the two ends too (Figure S 7C).
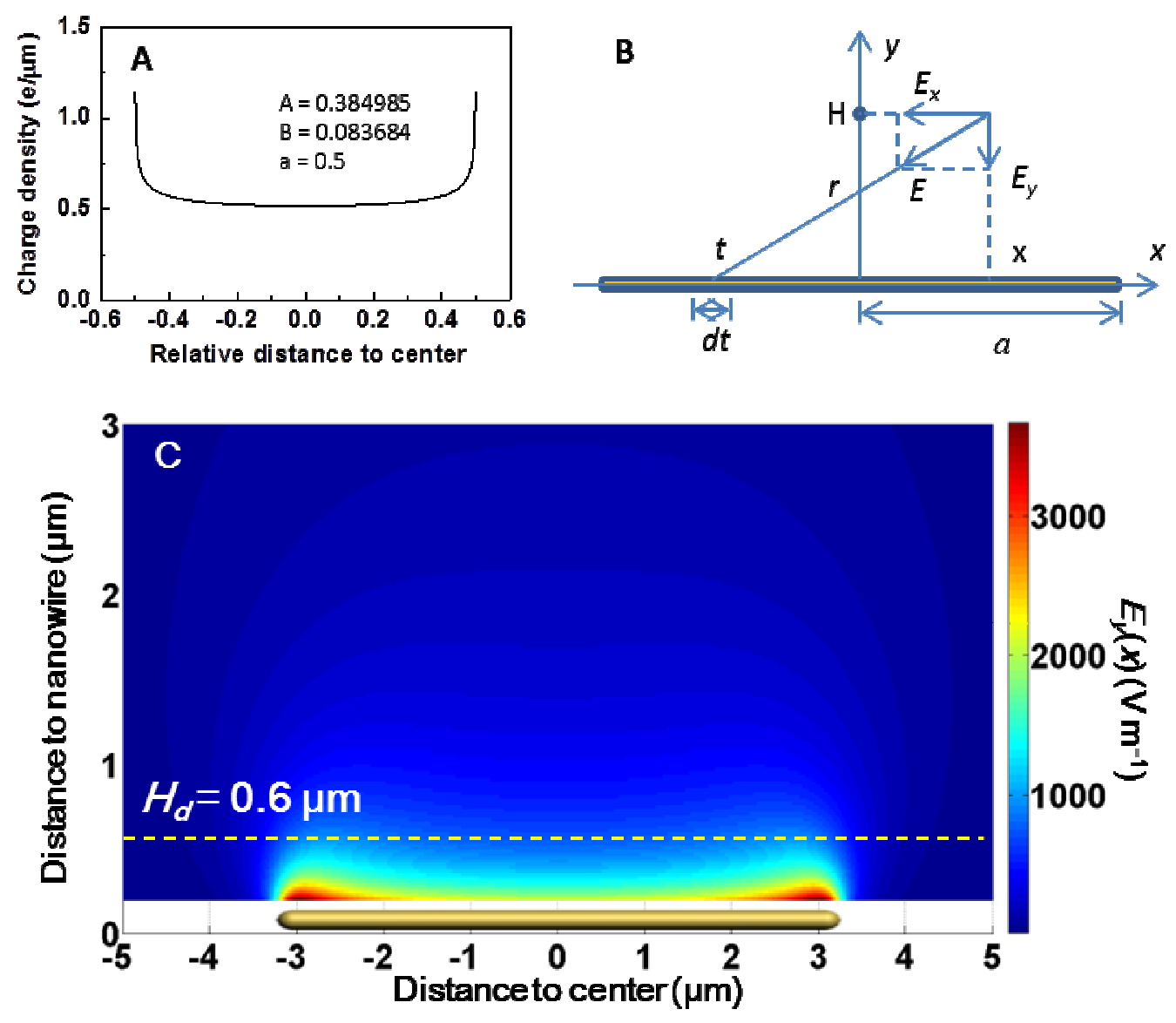

Figure S 7. (A) Simulation of charge distribution on a needle by Eq. S5. (B) Calculation of the intensity of electric field in y direct around the needle. (C) Intensity of electric field in y direct around the needle. Assume there are 100 electrons on the Ag nanowire.

At steady state, the reduction reaction rate of $\mathrm{Ag}^{+}$and $\mathrm{AuCl}_{4}^{-}$is, 
$r_{A g^{+}}(x)=\mu_{A g^{+}} c_{A g^{+}}^{H} E_{y}(x)-D_{A g^{+}} \frac{d c_{A g^{+}}}{d h}$

$r_{A u C l_{4}^{-}}(x)=-\mu_{A u C l_{4}^{-}} c_{A u C l_{4}^{-}}^{H} E_{y}(x)-D_{A u C l_{4}^{-}} \frac{d c_{A u C l_{4}^{-}}}{d h}$

So the total reaction rate is,

$r(x)=r_{A g^{+}}(x)+r_{A u C l_{4}^{-}}(x)$

$r(x)=\left(\mu_{A g^{+}} c_{A g^{+}}^{H} E_{y}(x)-D_{A g^{+}} \frac{d c_{A g^{+}}}{d h}\right)+\left(-\mu_{A u C l_{4}^{-}} c_{A u C l_{4}^{-}}^{H} E_{y}(x)-D_{A u C l_{4}^{-}} \frac{d c_{A u C l_{4}^{-}}}{d h}\right)$

$r(x)=\left(\mu_{A g^{+}} c_{A g^{+}}^{H}-\mu_{A u C l_{4}^{-}} c_{A u C l_{4}^{-}}^{H}\right) E_{y}(x)-D_{A g^{+}} \frac{d c_{A g^{+}}}{d h}-D_{A u C l_{4}^{-}} \frac{d c_{A u C l_{4}^{-}}}{d h}$

If the scattering intensity has a linear relationship with the reaction rate,

$\frac{\Delta I_{A g}(x)}{\Delta t}=k_{A g} r_{A g^{+}}(x)$
$\frac{\Delta I_{A u}(x)}{\Delta t}=k_{A u} r_{A u C l_{4}^{-}}(x)$

Then the scattering intensity of Ag nanowire at the location $x$ is,

$r^{\prime}(x)=\frac{\Delta I(x)}{\Delta t}=\frac{\Delta I_{A g}(x)}{\Delta t}+\frac{\Delta I_{A u}(x)}{\Delta t}=k_{A g} r_{A g^{+}}(x)+k_{A u} r_{A u C l_{4}^{-}}(x)$

Where $k_{A g}$ and $k_{A u}$ are the relationship between the scattering intensity and the deposition reaction rate for $\mathrm{Ag}$ and $\mathrm{Au}$. We use these equations to fit the data in Figure 6. In the main text, we show that these equations can fit the experimental results very well in Figure 6. But the fitting only give the concentration dependent thickness of diffusion layer. The more interesting parameter, i.e. total charge on nanowire, cannot be obtained, because the parameters $k_{A g}$ and $k_{A u}$ are not known. It should be mentioned that this model is suitable for the statistic results, such as the average reaction rate. 


\section{SI.6. More Discussion}

Besides the effect of net surface charge, some other commonly possible reasons including the facets, defects, surfactant, surface plasmon resonance, post-ripening process and diffusion are considered. First, if the reason is due to the facets or surfactant, the activity along the nanowire should be similar, since the wall of the nanowire has the same facet $\left((100)^{8}\right)$ and is homogeneously covered by surfactant. But the research shows that the activity, i.e. the appearance of bright spots, is unevenly distributed in Fig. 2. Second, if the reason is due to the defects, the center of nanowire should have higher activity, since the center has more defects. ${ }^{9}$ But the two ends of nanowire show higher activity during GRR at low $\left[\mathrm{HAuCl}_{4}\right]$ in this research (Fig. 2A). Third, if the reason is due to the surface plasmon resonance ${ }^{10}$, the activity distribution should be different with/without light emission. But Fig. 2A and Fig. 3A show that the activity distribution doesn't charge under or not under DFM. Furthermore, the Ag nanowire is too long $(\sim 6 \mu \mathrm{m})$ to have surface plasmon resonance in longitudinal direction in the wavelength of visible light. Fourth, if the reason is due to the post-ripening process, the intensity of the whole nanowire should increase uniformly. This phenomenon indeed happens at high $\left[\mathrm{HAuCl}_{4}\right]$. $\mathrm{But}$ this process only affects the intensity of the whole nanowire, the distribution of bright spots is still not uniform at high $\left[\mathrm{HAuCl}_{4}\right]$. Fifth, if the reason is due to the diffusion, the two ends of nanowire should be always more active than the mid part for low and high $\left[\mathrm{HAuCl}_{4}\right]$ s. But Fig. 2D shows that the activity is higher in the mid part for high $\left[\mathrm{HAuCl}_{4}\right] \mathrm{s}$. As a conclusion of above discussion, the above factors are not possible to be the reason for the unevenly distributed activity at different $\left[\mathrm{HAuCl}_{4}\right] \mathrm{s}$.

It should be mentioned that $\mathrm{Ag}$ atoms are impossible to directly migrate from the body to the $\mathrm{Ag}$ cluster, since the nanowire is very long $(\sim 6.3 \mu \mathrm{m}$ in average) and reaction temperature is low (room temperature).

The literature from Zheng et. $\mathrm{al}^{11}$ shows that PVP on Pd nanowire can be removed by washing with ethanol. Au mainly deposits on the tip of Pd nanowire before washing, while Au will evenly deposit on Pd nanowire after washing. Washing Ag nanowire can remove the coverage of PVP on Ag nanowire. 


\section{Supplementary Information}

The generation of $\mathrm{AgCl}$ precipitate at low $\mathrm{AuCl}_{4}{ }^{-}$concentration is difficult. This is due to several reasons:

1. Since the Ag nanowires are sparsely spread on glass slide, the absolute weight is very small.

2. Except for the critical state or high $\mathrm{AuCl}_{4}{ }^{-}$concentration, $\mathrm{GRR}$ on $\mathrm{Ag}$ nanowire is relatively low.

3. The formation of Au shell on Ag nanowire surface, which inhibits further GRR.

4. The reactant solution was kept flowing during the reaction, and the reaction time is as long as 2 hours. The $\mathrm{Ag}^{+}$and $\mathrm{Cl}^{-}$are hard to accumulate to a high concentration in the flow cell at slow reaction rate.

5. In addition, although the solubility of $\mathrm{AgCl}$ is very low in water, the concentrations of $\mathrm{Ag}^{+}$and $\mathrm{Cl}^{-}$are too low to generate $\mathrm{AgCl}$ precipitate. At high reaction rate of $\mathrm{GRR}, \mathrm{AgCl}$ will form. Therefore, for low $\mathrm{AuCl}_{4}{ }^{-}$concentration, $\mathrm{AgCl}$ may form during the critical state at about $1700 \mathrm{~s}$ in Figure 2. The formation of $\mathrm{AgCl}$ will supply a large amount of electrons for the deposition of $\mathrm{Ag}^{+}$. For high $\mathrm{AuCl}_{4}{ }^{-}$concentration, $\mathrm{AgCl}$ will form at the beginning of GRR.
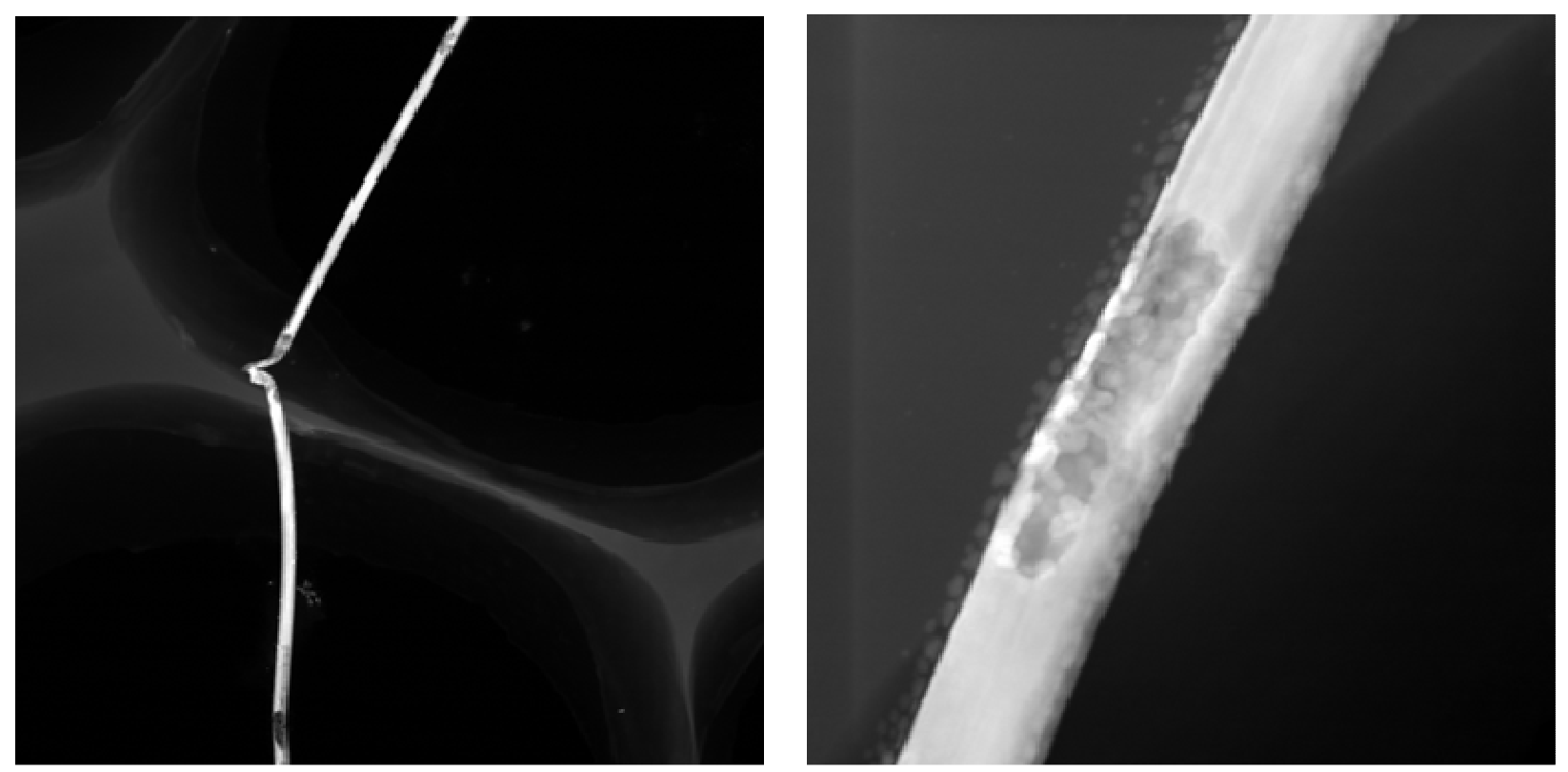

Figure S 8. STEM image of the pits formed on the surface of the nanowire. 
Supplementary Information

Table 1. Table of parameters

\begin{tabular}{|c|c|c|c|}
\hline Name of parameters & Symbol & value & Note \\
\hline Ion mobility of $\mathrm{Ag}^{+}\left(\mathrm{m}^{2} \mathrm{~s}^{-1} \mathrm{~V}^{-1}\right)$ & $\mu_{\mathrm{Ag}^{+}}$ & $6.42 \times 10^{-8}$ & 12 \\
\hline Ion mobility of $A u C l_{4}^{-}\left(\mathrm{m}^{2} \mathrm{~s}^{-1} \mathrm{~V}^{-1}\right)$ & $\mu_{A u C l^{-}}$ & $7.05 \times 10^{-8}$ & $\begin{array}{l}{ }^{12} \mathrm{Ion} \\
\mathrm{ClO}_{4}^{-}\end{array}$ \\
\hline Diffusion coefficient of $\mathrm{Ag}^{+}\left(\mathrm{m}^{2} / \mathrm{s}\right)$ & $D_{A g^{+}}$ & $1.648 \times 10^{-9}$ & 12 \\
\hline Diffusion coefficient of $A u C l_{4}^{-}\left(\mathrm{m}^{2} / \mathrm{s}\right)$ & $D_{A u C l^{-}}$ & $9.59 \times 10^{-10}$ & 12 \\
\hline Vacuum permeability $(\mathrm{F} / \mathrm{m})$ & $\varepsilon_{0}$ & $8.85 \times 10^{-12}$ & 12 \\
\hline Dielectric constant of solution & $\varepsilon$ & 87 & 12 \\
\hline Electron charge $(\mathrm{C})$ & $\mathrm{e}$ & $1.60 \times 10^{-19}$ & 12 \\
\hline Boltzmann constant $(\mathrm{J} / \mathrm{K})$ & $\mathrm{k}$ & $1.38 \times 10^{-23}$ & 12 \\
\hline Number of electrons & $\mathrm{Q}$ & counts & \\
\hline Temperature $(\mathrm{K})$ & $\mathrm{T}$ & 293 & \\
\hline Electric field in y direction $\left(\mathrm{V} \mathrm{m}^{-1}\right)$ & $E_{y}(x)$ & - & Variable \\
\hline Distance to nanowire (m) & $H$ & - & Variable \\
\hline Charge density at the position $\mathrm{x}\left(\mathrm{m}^{-1}\right)$ & $\sigma(x)$ & - & Variable \\
\hline Concentration of $\mathrm{Ag}^{+}\left(\mathrm{mol} \mathrm{m}^{-3}\right)$ & $c_{A g^{+}}$ & - & Variable \\
\hline Concentration of $\mathrm{AuCl}_{4}^{-}\left(\mathrm{mol} \mathrm{m}{ }^{-3}\right)$ & $c_{\mathrm{AuCl}_{4}^{-}}$ & - & Variable \\
\hline
\end{tabular}

\section{References:}

(1) Smith, J. G.; Yang, Q.; Jain, P. K. Identification of a Critical Intermediate in Galvanic Exchange Reactions by Single-Nanoparticle-Resolved Kinetics. Angew. Chem. Int. Ed. 2014, 53, 2867-2872.

(2) Bard, A. J.; Faulkner, L. R., Electrochemical Methods: Fundamentals and Applications, 2 nd Edition. John Wiley \& Sons Inc.: 2001.

(3) Zhao, J.; Huang, J.-Q.; Wei, F.; Zhu, J. Mass Transportation Mechanism in Electric-Biased Carbon Nanotubes. Nano Lett. 2010, 10, 4309-4315. 
(4) Bologa, M. K.; Grosu, F. P. Enhancement of Heat and Mass Transfer by an Electric Field. Surf. Engin. Appl.Electrochem. 2012, 48, 456-464.

(5) Zhang, L.; Henkelman, G. Tuning the Oxygen Reduction Activity of Pd Shell Nanoparticles with Random Alloy Cores. J. Phys. Chem. C 2012, 116, 20860-20865.

(6) Gao, H.; Liu, C.; Jeong, H. E.; Yang, P. Plasmon-Enhanced Photocatalytic Activity of Iron Oxide on Gold Nanopillars. Acs Nano 2012, 6, 234-240.

(7) Griffiths, D. J.; Li, Y. Charge Density on a Conducting Needle. Am. J. Phys. 1996, 64, 706-714.

(8) Sun, Y.; Mayers, B.; Herricks, T.; Xia, Y. Polyol Synthesis of Uniform Silver Nanowires: A Plausible Growth Mechanism and the Supporting Evidence. Nano Lett. 2003, 3, 955-960.

(9) Zhou, X.; Andoy, N. M.; Liu, G.; Choudhary, E.; Han, K.-S.; Shen, H.; Chen, P. Quantitative Superresolution Imaging Uncovers Reactivity Patterns on Single Nanocatalysts. Nat. Nanotechnol. 2012, 7, $237-241$.

(10) Link, S.; El-Sayed, M. A. Shape and Size Dependence of Radiative, Non-radiative and Photothermal Properties of Gold Nanocrystals. Int. Rev. Phys. Chem. 2000, 19, 409-453.

(11) Huang, X.; Zheng, N. One-Pot, High-Yield Synthesis of 5-Fold Twinned Pd Nanowires and Nanorods. J. Am. Chem. Soc. 2009, 131, 4602-4603.

(12) Lide, D. R., Ed. CRC Handbook of Chemistry and Physics; 76th ed. CRC Press, Inc.: 1995. 